\title{
Genome editing through large insertion leads to the skipping of targeted exon
}

Borhan Uddin ${ }^{1,2+}$, Nan-Peng Chen ${ }^{1,2+}$, Marko Panic ${ }^{1,2}$ and Elmar Schiebel ${ }^{1 *}$

\begin{abstract}
Background: Highly efficient genome editing can be achieved through targeting an endonuclease to specific locus of interest. Engineered zinc-finger nuclease (ZFN) and CRISPR-associated protein-9 nuclease (Cas9) offer such an elegant approach for genome editing in vertebrate cells. In this study, we have utilized ZFN and Cas9-catalyzed double strand break followed by homologous recombination-mediated incorporation of premature stop codon and selection marker to target human cell division cycle 14A ( $h C D C 14 A)$ and cell division cycle 14B ( $h C D C 14 B)$ genes.

Results: Targeting of the $h C D C 14 A$ and $h C D C 14 B$ loci in telomerase immortalized human retinal pigment epithelium (hTERT-RPE1) and human colon cancer (HCT116) cells were confirmed by Southern blot hybridization. Nevertheless, DNA sequence analysis of reverse transcription polymerase chain reaction (RT-PCR) products confirmed that in all the single/double allele ablations, the targeted exon was spliced out. The phenomenon of exon skipping was independent of the genome editing approaches exploited, Cas9 or ZFN. Because the exons had a nucleotide number that could be divided by 3 , the reading frame of the exon deletion was maintained. This indicates an exon-skipping event possibly due to the insertion of large DNA fragment (1.7 to $2.5 \mathrm{~Kb}$ ) within the targeted exons. As a proof-of-principle, we have used gene disruption followed by non-homologous end joining (NHEJ) approach. Small alterations in the exon (one to fifteen bases) were transcribed to mRNA without exon skipping. Furthermore, loxP site-mediated removal of selection markers left a 45 bp scar within the targeted exon that can be traced in mRNA without exon skipping.

Conclusion: From this study, we conclude that insertion of a large DNA fragment into an exon by genome editing can lead to its skipping from the final transcript. Hence, more cautious approach needs to be taken while designing target sites in such that the possible skipping of targeted exon causes a frame-shift mediated incorporation of pre-mature stop codon. On the other hand, exon skipping may be a useful strategy for the introduction of protein deletions.
\end{abstract}

Keywords: Genome engineering, Zinc-finger nucleases, CRISPR-Cas9, Exon-skipping, hCDC14A, hCDC14B

\section{Background}

Highly dynamic, yet tightly controlled, protein phosphorylation and de-phosphorylation events are important avenues to tweak cell cycle transition processes. Cyclin-dependent kinases (CDKs) lie at the heart of cell cycle control system and their activities rise and fall as the cell progresses through the cell cycle (Reviewed in [1]). In budding yeast Saccharomyces cerevisiae, the highly conserved ScCdc14 (cell division cycle 14 gene)

\footnotetext{
* Correspondence: e.schiebel@zmbh.uni-heidelberg.de

${ }^{\dagger}$ Equal contributors

'Zentrum für Molekulare Biologie der Universität Heidelberg (ZMBH),

DKFZ-ZMBH Allianz, Im Neuenheimer Feld 282, 69120 Heidelberg, Germany

Full list of author information is available at the end of the article
}

phosphatase antagonizes the Cdk1 functions to allow anaphase regulation and mitotic exit [2]. Human cells encode three paralogs of $h C D C 14$ namely $h C D C 14 A$, $h C D C 14 B$ and $h C D C 14 C[3,4]$. In spite of the high conservations between the catalytic domain of all CDC14 phosphatases [5] and the complementation of $S c C d c 14$ by $h C D C 14 B[6]$, human $C D C 14$ s have so far been reported to be involved in functions that are quite diverse than that of budding yeast [7]. Human hCDC14A was proposed to exert its function at centrosome duplication [8] while hCDC14B was implicated in mitotic progression [9], DNA damage checkpoint activation and DNA repair [10]. Nevertheless, hCDC14B depleted human cells display normal mitotic exit and cytokinesis [11]. 
Moreover, the viability of $h C D C 14 A$ or $h C D C 14 B$ single knockout $(\mathrm{KO})$ vertebrate cells [12] indicate the possible functional redundancy of vertebrate phosphatases. It is noteworthy that most of the previously reported functions of hCDC14A/B were deduced upon siRNA depletion (often without a rescue experiment) or strong overexpression that causes toxic effects. Extent of depletion as well as the functional redundancy of the phosphatases was not taken into consideration partly because of the inability of available antibodies to recognize endogenous hCDC14A and hCDC14B proteins [12-14].

Genome editing provides an alternative strategy to siRNA depletion for hCDC14A and hCDC14B inactivation. Currently, several strategies exploiting sequencespecific endonucleases exist for purposeful genome editing, including Zinc-finger nuclease (ZFN), transcription activator-like effector nuclease (TALEN), and the RNAguided clustered regularly interspaced short palindromic repeats (CRISPR)-Cas9 nuclease system [15]. In all these approaches, an endonuclease is programmed to specifically bind the assigned nucleotide sequence and trigger single or double strand breaks (DSB). Cells can take either high-fidelity homologous recombination (HR) and/or error-prone non-homologous end-joining (NHEJ) to repair this DSB [16] (Additional file 1: Figure S1). NHEJ might alter the DSB site by random insertion or deletion of nucleotides of varying length. On the other hand, homologous recombination can be utilized to deliberately introduce stop codons and selectable markers for ensuring genome disruption (Additional file 1: Figure S1).

In this study, we have used DSB-enhanced HR event to incorporate pre-mature stop codons followed by selection markers ( $\sim 2 \mathrm{~kb}$ sizes) within the selected exons of human $h C D C 14 A$ and $h C D C 14 B$ genes. Nonetheless, Cas9 and ZFN-mediated genome disruption strategies have unfolded complications owing to the in-frame skipping of targeted exons. We presume that interruption due to the incorporation of a large fragment of DNA (1.7 to $2.5 \mathrm{~kb}$ including stop codon and selection markers) has structural effects on exon definition as reviewed by Valentine [17]. LoxP sitemediated removal of selection markers from the previously generated knockins prevented exon skipping but left a 45 bp Stop-loxP scar within the targeted exon. Thus, we report that insertion of large DNA fragment into an exon by genome editing leads to its skipping from the final transcript and how this property can be used in genome editing.

\section{Results and discussion}

\section{Disruption of the $h C D C 14 A$ and $h C D C 14 B$ loci in human} somatic cells

We have utilized CompoZr ${ }^{\mathrm{TM}}$ ZFNs, designed and evaluated by Sigma Advanced Genetic Engineering Labs, to make $h C D C 14 A$ and $h C D C 14 B$ knockouts in two human cell lines (hTERT-RPE1 and HCT116) with stable genotype. ZFN-induced homologous recombination strategy was employed to incorporate premature stop codon and selection markers (neomycin or puromycin) into the targeted exons of $h C D C 14 A$ ( $9^{\text {th }}$ exon) and $h C D C 14 B\left(4^{\text {th }}\right.$ exon) (Fig. 1a, b). Southern blot analysis has confirmed the successful biallelic targeting of the $h C D C 14 A$ and $h C D C 14 B$ loci by NeoR or PuroR cassettes (Fig. 2a-c).

\section{RT-PCR analysis confirming expression of wild type and in-frame exon-skipped $h C D C 14 A / h C D C 14 B$ transcripts} Sequencing of the $h C D C 14 A$ and $h C D C 14 B$ RT-PCR (reverse transcriptase polymerase chain reaction) products from wild type and selection marker knockins verified the presence of exon-skipped mature mRNA in the targeted hCDC14A and hCDC14B clones (Fig. 3a, b). The reading frames remain intact as the skipped exons in both the cases $\left(9^{\text {th }}\right.$ exon for hCDC14A and $4^{\text {th }}$ exon for hCDC14B) contained nucleotide number that could be divided by three. Hence, we have to assume the presence of truncated $\mathrm{hCDC} 14 \mathrm{~A}$ and $\mathrm{hCDC} 14 \mathrm{~B}$ proteins.

The $9^{\text {th }}$ exon of hCDC14A includes the active site cysteine and aspartate residues necessary for phosphatase activity $[5,18,19]$, suggesting the inactivation of hCDC14A phosphatase from these cell lines. Conversely, the catalytically important residues for $\mathrm{hCDC} 14 \mathrm{~B}$ are located in $9^{\text {th }}$ exon, downstream to the targeted exon [5]. Thus, in case of $\mathrm{hCDC} 14 \mathrm{~B}$ the gene interference strategy most likely creates a small truncation of 31 amino acids in the hCDC14B protein that might not affect phosphatase activity.

\section{Exon-skipping phenomenon is independent of genome editing approach}

Our ZFN-mediated genome editing of $h C D C 14 A$ and $h C D C 14 B$ has clearly indicated the skipping of targeted exon from the final transcript. As a faster and more affordable alternative to $\mathrm{ZFN}$, there is a recent surge in use of CRISPR-Cas9 system for genome editing. We have taken the advantage of CRISPR-Cas9 system to target $h C D C 14 A$ locus with the same donor construct and guide RNA that targets the same genomic $h C D C 14 A$ DNA sequence as the ZFN. As the lengthy custom design of the ZFN was avoided, CRISPR-Cas9 strategy was clearly faster and more than 50-fold cost-efficient than the ZFN approach. As anticipated, we observed exon skipping in HCT116 and RPE1 cells in which $h C D C 14 A$ was targeted by CRISPR-Cas9 (Fig. 4, Additional file 2: Figure S2). This further implies that the skipping event is associated with the degree of exon alteration not merely a random outcome of genome editing approach. 


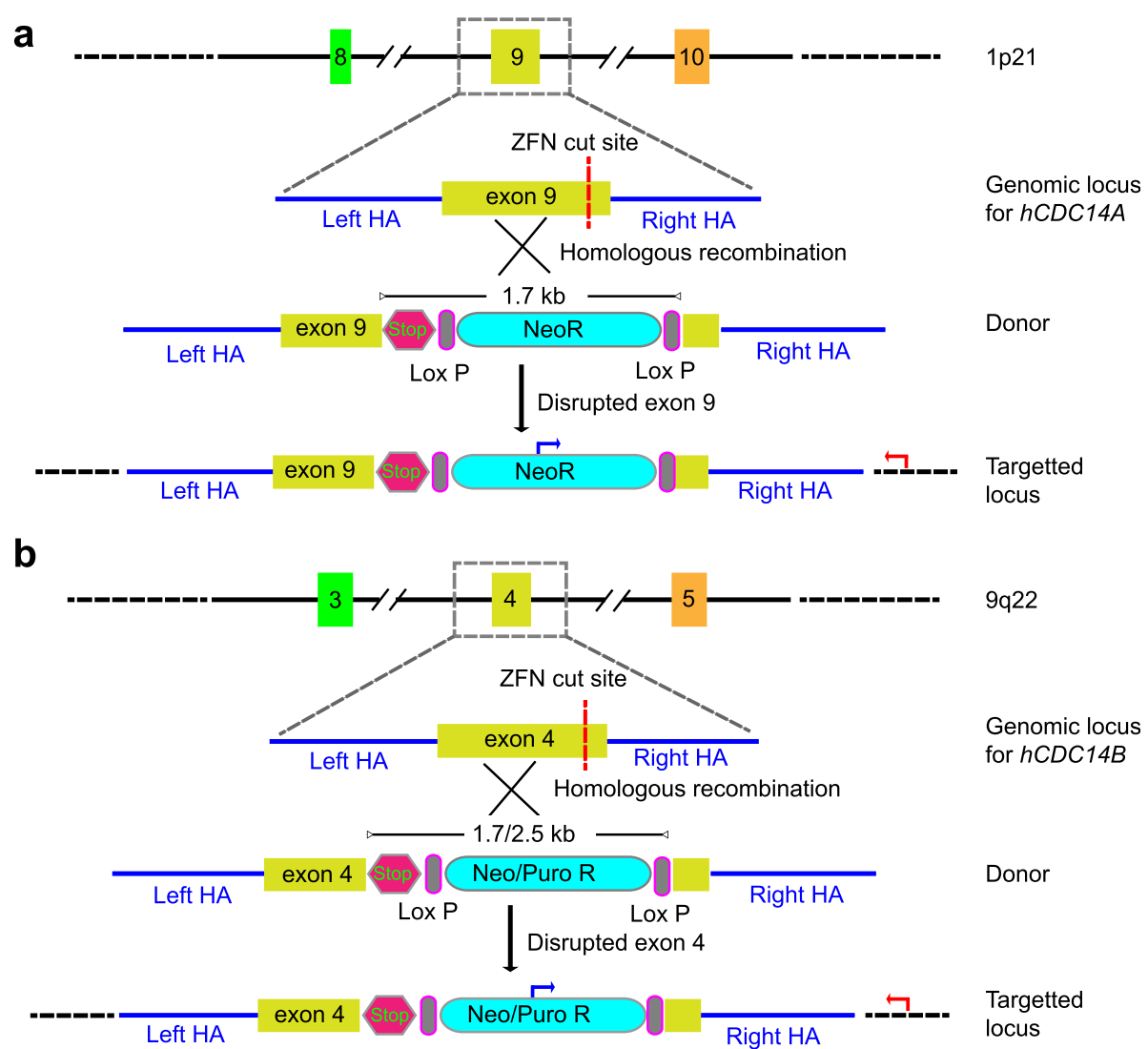

Fig. 1 Strategy for ZFN-mediated generation of knockout cell lines. a Exon 9 of $h C D C 14 A$ was targeted by zinc finger nuclease (ZFN). A donor template containing two homologous arms (HA), stop codon and neomycin selection cassette was used for their homologous recombination mediated insertion within the double strand break (DSB) site. Junction PCR with forward primer (blue arrow) in NeoR cassette and reverse primer (red arrow) in the genome outside homology arm confirmed successful targeting and insertion of the selection marker. $\mathbf{b}$ Exon 4 was targeted to knockout $h C D C 14 B$ gene. As donor template for $h C D C 14 A$, neomycin selection cassette was used to generate $h C D C 14 B$ single knockout. Puromycin cassette was used when $h C D C 14 B$ was knocked-out on top of $h C D C 14 A^{-1-}$ cells (neomycin). Forward (blue) and reverse (red) primers used for the junction PCR are shown in the figure

\section{Minimizing the degree of alteration salvages the exon skipping}

As a cause of exon skipping, we hypothesized that large insertion-mediated alteration of the targeted exon affects the pre-mRNA structure [17]. Hence, to avoid largescale genome editing, we have taken two experimental strategies. First, genomic loci were targeted by the same ZFNs but with error-prone NHEJ, with the view in mind to have random smaller insertions or deletions (Fig. 5a). Secondly, the selection markers flanked by loxP sites were removed by Cre-recombinase to generate loci with 45 base pair insertions including stop codons immediately followed by the single loxP site (Fig. 5b). In both the cases, sequencing of genomic loci indicated the expected abruptions. Similarly, sequencing of the RT-PCR products confirmed the likewise modifications within the targeted exons without their skipping from the mature transcript (Fig. 5a, b).

Semi-quantitative RT-PCR using GAPDH as a control indicated that the exon-skipped $h C D C 14 B$ mRNA level was less than half of the wild-type mRNA (Fig. 5c). Such decrease in mRNA level might be due to transcriptional control (altered synthesis) and/or post-transcriptional regulation (altered stability) of disrupted cellular transcripts. Because of the lack of anti-hCDC14B antibodies that detect the endogenous proteins [12-14], we were unable to confirm this mRNA decrease at the protein levels.

Taken together, we describe that insertion of a relatively large DNA fragment into an exon can cause exon skipping. If the exon has a length that can be divided by 3 , this leads to an in-frame fusion between the alternating exons. In case of $\mathrm{hCDC} 14 \mathrm{~A}$, the truncated gene product lacks phosphatase activity because the deleted exon 9 contains the active site cysteine residue. Since phosphatases can have a scaffold function [20], analysis of this hCDC14A-Cys mutant can be used to uncover such a role. In contrast, deletion of the exon 4 of hCDC14B most likely results in a small truncation in the $\mathrm{N}$-terminus of hCDC14B away from the nucleolar targeting sequence [7]. Since the residues required for 


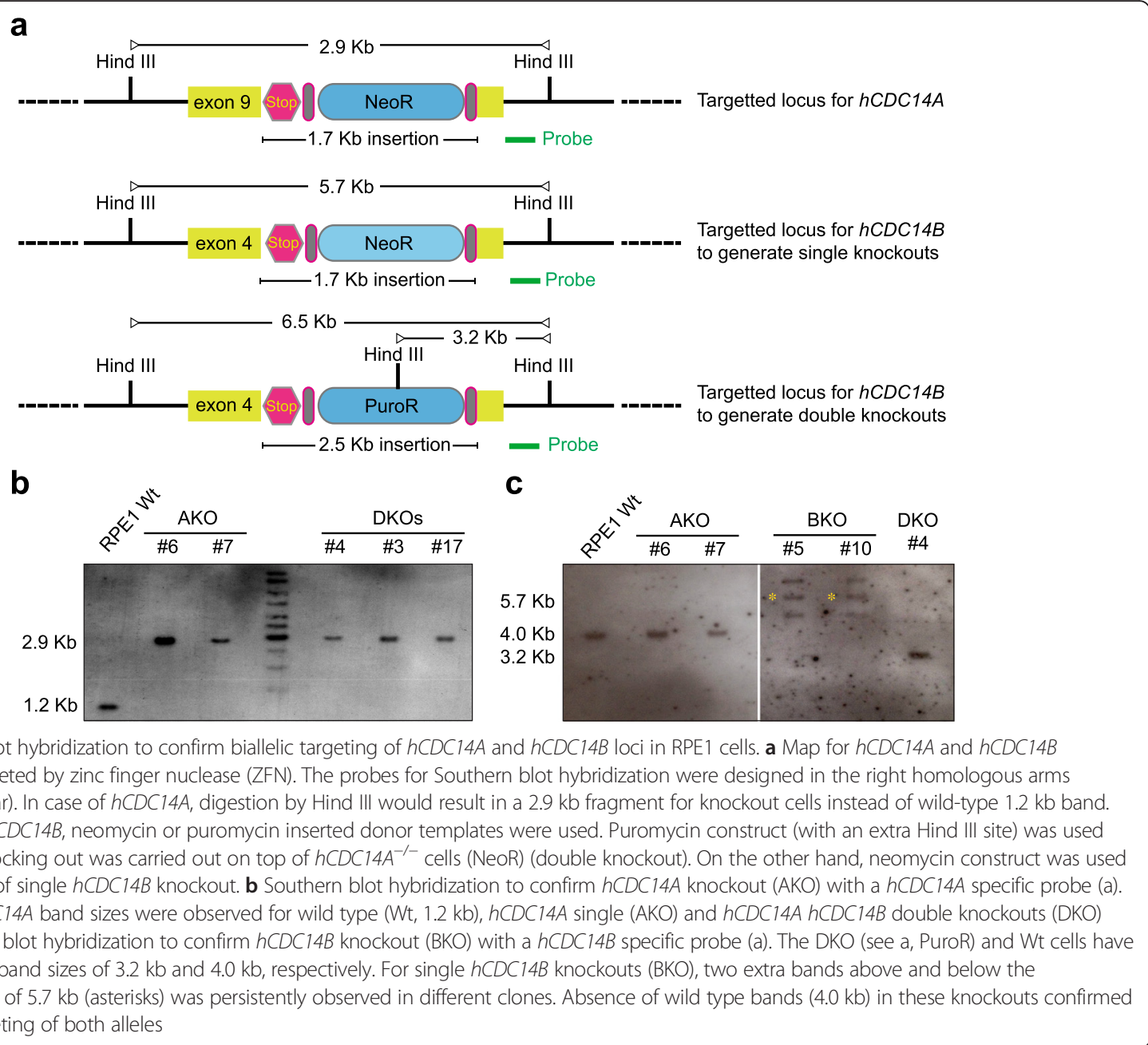

hCDC14B activity were not affected, it is likely that this truncation does not affect the activity of the phosphatase. However, removal of the selection marker by Cre-induced recombination prevented exon skipping and disrupted gene function by introducing stop codons. Thus, adenovirus-Cre mediated infection of hCDC14B::neomycin cells can be used to introduce an acute knockout of $\mathrm{hCDC14B}$ function.

\section{Conclusion}

Genome editing is a robust experimental paradigm to assign cellular function(s) to a molecule. Simplicity of genome editing through engineered nucleases has recently allured the scientific community to generate knockout cellular models. Utilizing selection markers greatly augments the ease of generating knockout cellular clones. However, our study clearly shows that, the selection marker also imposes an inherent size-associated mRNA processing difficulty of the targeted exon. In most of the cases, the selection markers overweigh the average length of eukaryotic exon $(<200 \mathrm{bp},[21,22])$. For example, the $4^{\text {th }}$ exon of $h C D C 14 B$ is 93 bases long compared to the $1.7 \mathrm{~kb}$ of neomycin and $2.5 \mathrm{~kb}$ of puromycin cassette. Such massive alterations have the potential to annihilate secondary pre-mRNA structure and impede cellular mRNA processing leading to exon skipping. Well-informed and pre-planned experimental choices should be made to target the suitable exon while editing the genome of higher eukaryotes like in human cells. An alternative strategy could be the introduction of double strand break in an intronic sequence to incorporate homologous recombination mediated subtle changes in the adjacent exon. These exon modifications could be introduced in the donor along with the selection marker. Of note, such skipping mechanisms can be useful to engineer proteins with small deletions or for the analysis of acute knockouts in response to Cre - induced selection marker removal.

\section{Methods}

Generation of ZFN-mediated knockout cells

Human CDC14A and CDC14B specific CompoZr ${ }^{\mathrm{rm}}$ knockout Zinc Finger Nucleases were designed and evaluated by 


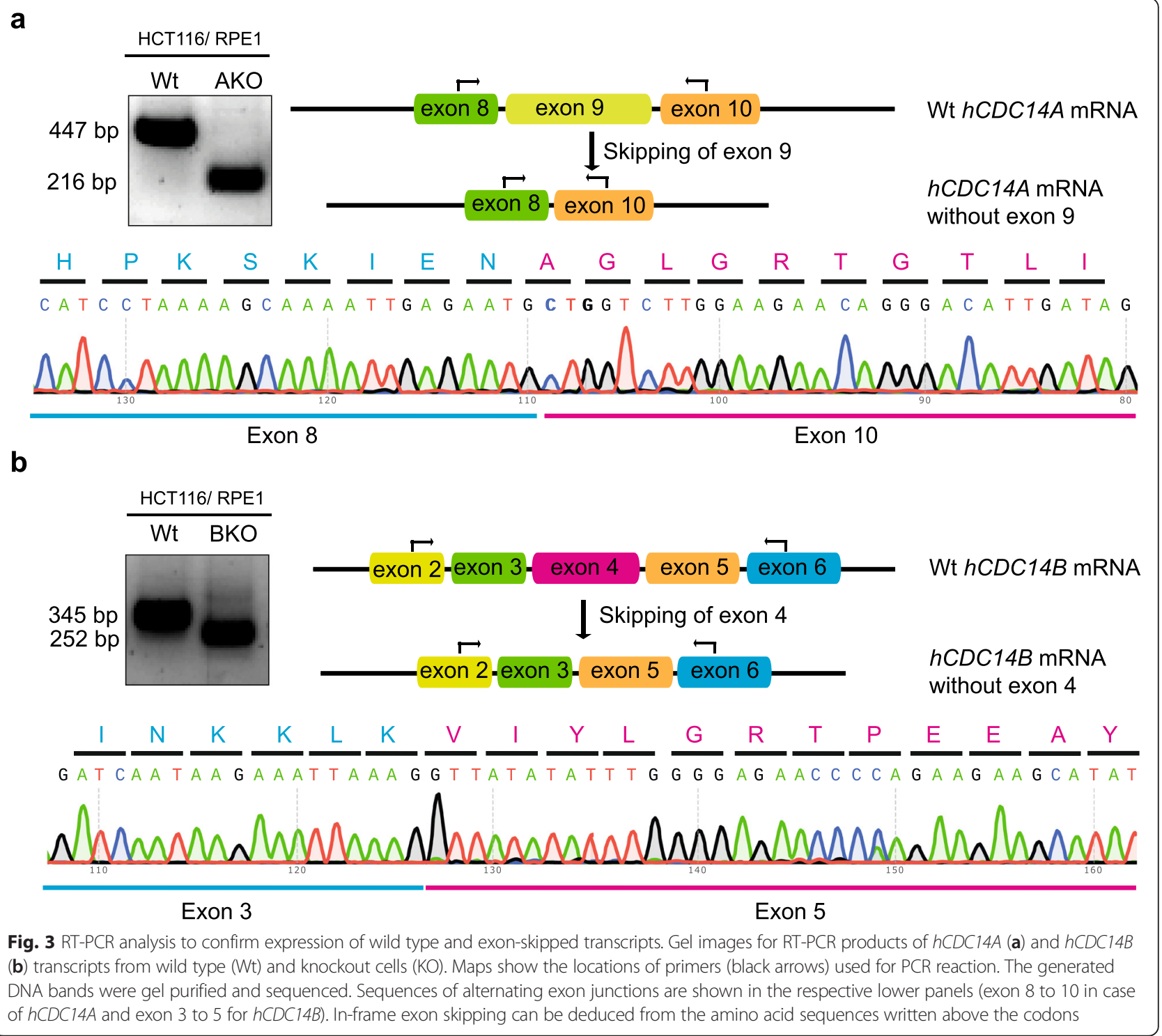

Sigma Advanced Genetic Engineering Labs. The ZFN for $h C D C 14 A$ (product Number: CKOZFND2170-1KT) targets the sequence $5^{\prime}$-AGCACACCCAGTGACaacatCGT GCGAAGGTTCCTGAA-3' in the 9th exon (cutting site in lower cases). Target sequence for ZFN against $h C D C 14 B$ is in the 4th exon $5^{\prime}$-TGCTGCCTTCCTTGTtggatGCTA CATGGTAAGTATTTG-3' (product Number: CKOZFN D5769-1KT).

The donor vector was constructed by PCR amplification of the genomic locus as reported by Panic et al. [23]. In short, the genomic locus 800 bp upstream and downstream of the ZFN cut site was amplified and subcloned into pJet 1.2 vector. The insertion cassette including STOP codon in every frame and neomycin/ puromycin resistance was inserted at the cut site of donor vector.
$10^{6}$ cells were co-transfected with the ZFN mRNAs $(2.5 \mu \mathrm{g}$ of each) and donor vector $(7 \mu \mathrm{g})$ by electroporation (Invitrogen, Neon transfection system). After electroporation, cells were cultured for $24 \mathrm{~h}$ at $37{ }^{\circ} \mathrm{C}$ (recovery) followed by $48 \mathrm{~h}$ at $30{ }^{\circ} \mathrm{C}$ (enhancing ZFN efficiency [24]). Cells were further cultured at $37{ }^{\circ} \mathrm{C}$ for $72 \mathrm{~h}$ prior to single cell dilution (limiting) in 96 well plates $(500 \mu \mathrm{g} / \mathrm{ml}$ neomycin). Two weeks later, the emerging clones were screened by genomic PCR and positive clones were further confirmed by RT-PCR as well as southern blot hybridization. Junction PCR with one primer in donor construct and the other in the genome outside homology arm confirmed successful targeting and insertion of the selection markers. The following primers were used for junction PCR - hCDC14A: forward 5'-CGGCTATGACTGGGCACAAC-3'; reverse 


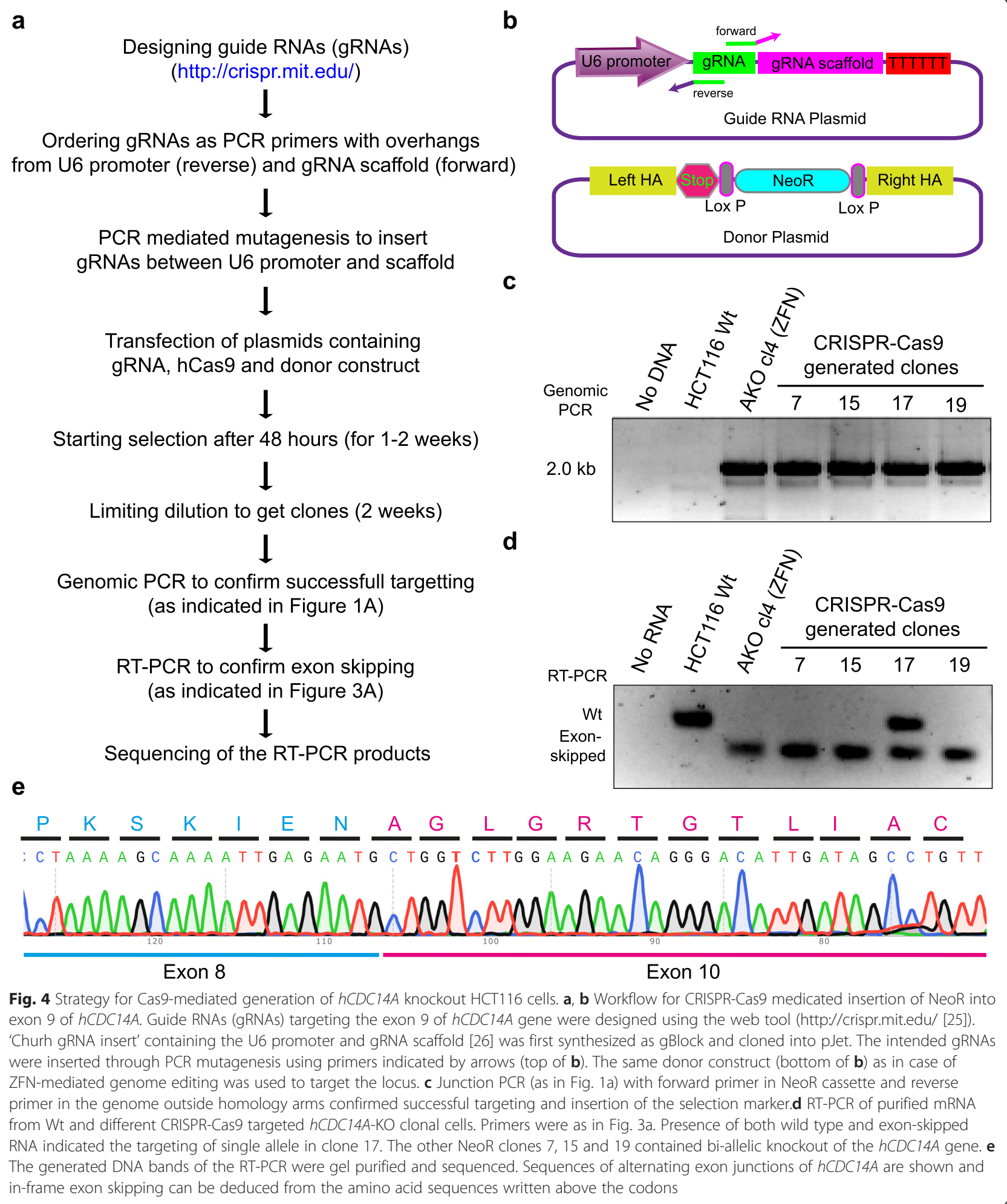

5' - GCCTCCTCGAAGTCAAACAAG -3'; hCDC14B (for NeoR insertion): forward 5'-CGGCTATGACTGGG CACAAC-3'; reverse 5'-CGATCTCCGCTCACTG-3'; hCDC14B (for PuroR insertion): forward 5' -CGGGGCG
AAGGCAAC-3'; reverse 5' -CGATCTCCGCTCACTG-3' . Similar approach without the donor construct was taken in case of NHEJ-facilitated disruption of $h C D C 14 B$ gene. The resultant insertion/deletion-mediated mutagenesis was 
a

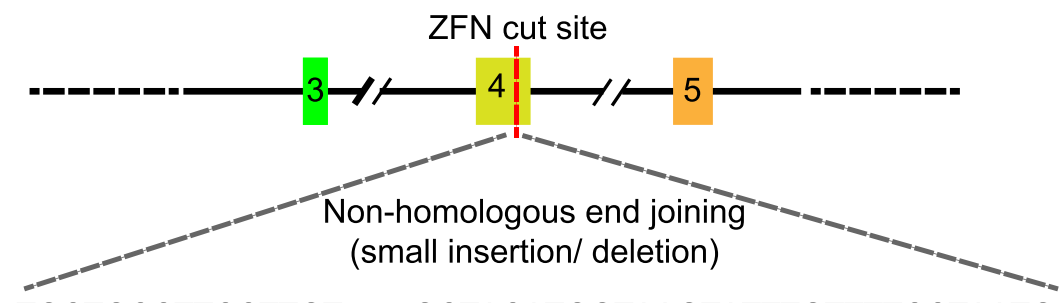

Genomic locus for $h C D C 14 B$

TGCTGCCTTCCTTGTtggatGCTACATGGTAAGTATTTGTTTTCCTAATG

HCT116 wild type

TGCTGCCTTCCTTGTtg-atGCTACATGGTAAGTATTTGTTTTCCTAATG

one $\mathrm{G}$ deletion

TGCTGCCTTCCTTGT-ggatGCTACATGGTAAGTATTTGTTTTCCTAATG

one $T$ deletion

TGCTGCCTTCCTTGTtggatTGGATGCTACATGGTAAGTATTTGTTTTCCTAATG

5 bp insertion

TGCTGCCTTCCTTGT-

AAGTATTTGTTTTCCTAATG

$\perp$

15 bp deletion

Similar changes (insertion/deletion) in mRNA, no exon skipping

b

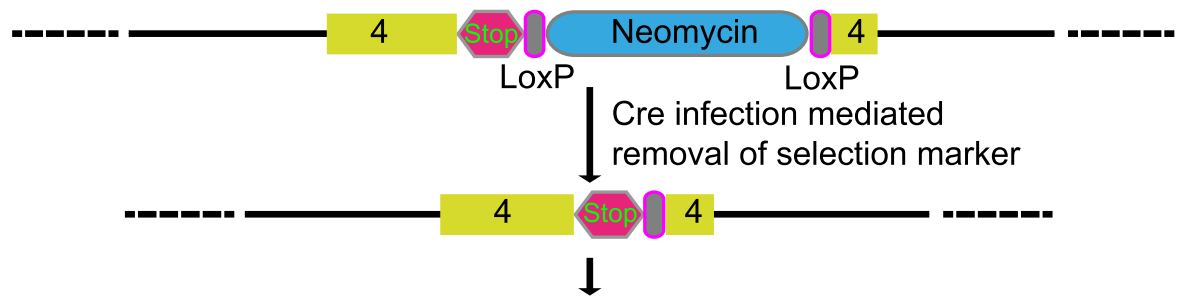

Targetted locus for $h C D C 14 B$ (HCT116/ RPE1)

Locus with stop codon and LoxP (HCT116/ RPE1)

Similar changes in mRNA (with stop codon and LoxP); No exon skipping
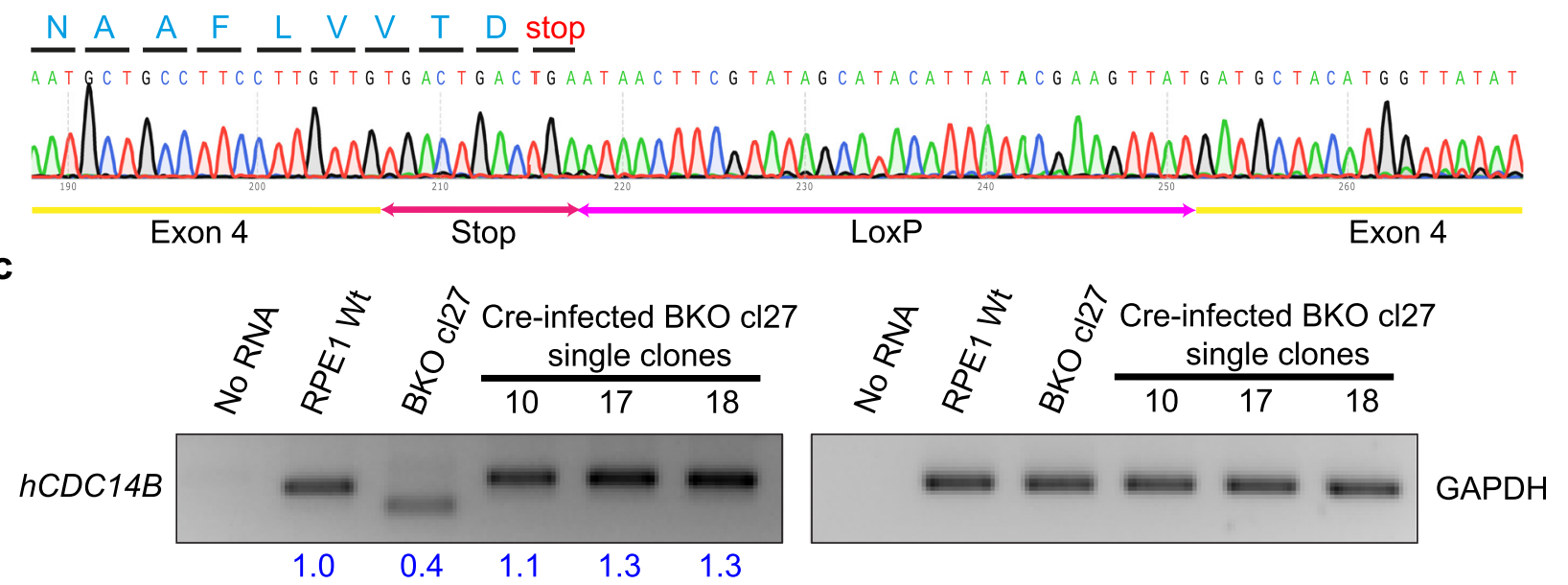

Fig. 5 Exon skipping can be abolished by minimizing the degree of alteration. a Exon 4 of hCDC14B was targeted by ZFN to introduce double strand break (DSB) and error-prone NHEJ for random insertion and deletion. In some of the targeted loci, small alterations in the exon (one to fifteen bases) were observed. ZFN cutting sites are written in blue font and base deletions or insertions are marked by red font color. Sequencing of the RT-PCR products confirmed identical base pair changes without exon skipping (sequence not shown). $\mathbf{b}$ The selection cassette flanked by loxP sites was removed by Cre-recombinase from HCT116 and RPE1 hCDC14B-KO cells. RT-PCR analysis confirmed the presence of stop codon (11 bp) followed by one loxP site (34 bp) within the targeted exon in both the cell lines. c Semi-quantitative RT-PCR indicated that the exon-skipped mRNA level of RPE1 cells is less than half of the wild-type mRNA. 40 ng of total RNA was used and the input RNA level was confirmed by GAPDH amplification as mentioned by Carbery et al. [30]. The experiment was performed three times with similar outcome. One representative experiment is shown. The numbers below the agarose gel summarizes the relative abundance of the mRNA from three different experiments

detected by sequencing of the amplified (Q5 ${ }^{\circ}$ High-Fidelity DNA Polymerase - NEB) genomic locus surrounding the ZFN cut site (primers used - forward 5' ${ }^{\prime}$ TGAATGGTTA TGGGATTTGGA-3'; reverse 5'-GCACAGCTTCCTTG AATTGG-3').
Generation of Cas9-mediated knockout cells Guide RNAs (gRNAs) targeting the exon 9 of $h C D C 14 A$ was designed using the web tool (http://crispr.mit.edu/ [25]). gRNA1 (5' -CCAGTGACAACATCGTGCGA-3'), gRNA2 (5'-CCTTCGCACGATGTTGTCAC-3') and gRNA5 
(5'-CTTCGCACGATGTTGTCACT-3') with scores 96, 91 and 86, respectively, were selected as they were exactly targeting the ZFN binding site (5'-AGCACACC CAGTGACaacatCGTGCGAAGGTTCCTGAA-3').

'Churh gRNA insert' containing the U6 promoter and gRNA scaffold [26] was first synthesized as gBlock from IDT (http://www.idtdna.com/pages/products/genes/gblocksgene-fragments) and cloned into pJet1.2 vector. The intended gRNAs were then ordered as PCR primers with overhangs from U6 promoter and gRNA-scaffold and inserted between U6 promoter and gRNA scaffold through PCR mutagenesis (Fig. 4).

Different strategies were used to generate $h C D C 14 A$ KO HCT116 and RPE1 cells. For HCT116, hCas9 plasmid (a gift from George Church (Addgene \# 41815, [26])) was transiently transfected with gRNA and donor plasmids. Whereas for RPE1 cells, pCW-Cas9 plasmid (a gift from Eric Lander \& David Sabatini (Addgene \# 50661 [27])) containing doxycycline (Dox) inducible spCas9 was lentivirally integrated into RPE1 FRT/T-Rex cells. Successful expression and nuclear localization of Cas9 was confirmed by indirect immunofluorescence and western blot analysis (Additional file 2: Figure S2). The cells were then electroporated with the plasmids containing gRNA and donor vector as showed in Fig. 4b. Junction PCR with forward primer in NeoR cassette (5'-CGGCTATGACTGGGCACAAC-3') and reverse primer in the genome outside homology arms (5'-GCCTCCTCGAAGTCAAACAAG-3') confirmed successful targeting and insertion of the selection marker.

\section{Southern blot hybridization to confirm knockouts}

Genome editing in both RPE1 and HCT116 cells was verified by Southern blot hybridization [28]. Genomic DNA was isolated using MasterPure DNA purification kit (Epicentre, Cat MCD85201) following manufactures instructions. $20 \mu \mathrm{g}$ of DNA were digested overnight with FastDigest Hind III-HF (Thermo Scientific) and run overnight onto a long $(18 \mathrm{~cm}) 0.8 \%$ agarose gel at $30-$ $35 \mathrm{~V}$. The gel was subsequently stained and photographed (fluorescent ruler of gel-casting tray was used to track the distance of migration of DNA bands). The gel was then washed in double distilled water $\left(\mathrm{ddH}_{2} \mathrm{O}\right)$ and DNA was denatured in $0.5 \mathrm{M} \mathrm{NaOH}, 1.5 \mathrm{M} \mathrm{NaCl}$ (twice for $20 \mathrm{~min}$ with gentle shaking). After rinsing once with $\mathrm{ddH}_{2} \mathrm{O}$, DNA was neutralized by washing thrice for $15 \mathrm{~min}$ with $1.5 \mathrm{M} \mathrm{NaCl}, 0.5 \mathrm{M}$ Tris- $\mathrm{HCl}$ (pH 7.0) and transferred onto a GeneScreen Plus ${ }^{\circ}$ Hybridization Transfer Membrane (PerkinElmer) by overnight capillary transfer in 10xSSC buffer $(1.5 \mathrm{M}$ $\mathrm{NaCl}, 0.15 \mathrm{M}$ trisodium citrate). After rinsing the membrane with 2XSSC buffer, DNA was UV-crosslinked with a Stratalinker 1800 (Stratagene).

The membrane was hybridized over night in DIG Easy Hyb buffer (Roche, cat\#11603558001) with DIG labeled probe generated by PCR DIG probe Synthesis kit (Roche, cat\#11636090910). Primers used to generate DIG probes for $h C D C 14 A$ : forward 5'-CATCGCCGTTCACTGC-3', reverse 5'-ACGTGGGCCTGGAAAG-3'; and $h C D C 14 B$ : forward 5'-GCCCAACTACTTTGGCAAAG-3', reverse 5' - CCAATGATCCAAATGGAGCAC-3'.

\section{RNA preparation from cells and expression analysis}

Total RNA was isolated from cells using the RNeasy Mini Kit (Qiagen, Hilden, Germany) following manufacturers protocol. RT-PCR analysis confirming expression of wild type and exon-skipped $h C D C 14 A / B$ transcripts were carried out using SuperScript ${ }^{\circ}$ III One-Step RTPCR System with Platinum ${ }^{\circ}$ Taq DNA Polymerase (Invitrogen). Forward primer binding to $8^{\text {th }}$ exon (5'ATGGTGACTTCAACTGGA-3') and reverse primer binding to $10^{\text {th }}$ exon $\left(5^{\prime}-C\right.$ TTCCAGGAAGTGCTGC$\left.3^{\prime}\right)$ were used for RT-PCR of $h C D C 14 A$ coding sequence (CDS). In case of $h C D C 14 B$, the forward primer was designed in the $2^{\text {nd }}$ exon ( $5^{\prime}$-GCCATTCTCTACAGCAG$\left.3^{\prime}\right)$ while reverse primer in $6^{\text {th }}$ exon $\left(5^{\prime}\right.$-GCAACTTCCATAGGCAGC-3'). Bands corresponding to wild type and exon-skipped clones were excised from the gel and sequenced to verify the presence of the splice junctions ( $h C D C 14 A$ : exon $8 \rightarrow 10$; $h C D C 14 B$ : exon $3 \rightarrow 5$ ). Human GAPDH was amplified with the primers described by Zhang et al. [29]: forward 5'-ATCCCATCACCATCTTCCAG-3' and reverse 5'-CCATCACGCCACAGTTTCC-3'. For semi-quantitative RT-PCR, $40 \mathrm{ng}$ of total RNA was used as template and the relative amount of mRNA in different samples was calculated by measuring the band intensity (normalized to GAPDH) using NIH ImageJ software [30] .

\section{Immunofluorescence microscopy and immunoblotting}

Cells were seeded on cover slips, allowed to attach and fixed with ice-cold methanol for $5 \mathrm{~min}$ at $-20{ }^{\circ} \mathrm{C}$ prior to permeabilization with $0.1 \%$ Triton X-100 for $10 \mathrm{~min}$. After, blocking with $10 \%(\mathrm{v} / \mathrm{v})$ fetal calf serum (FCS) for $30 \mathrm{~min}$, cells were stained with anti-bodies in $3 \%(\mathrm{w} / \mathrm{v})$ BSA (bovine serum albumin) in PBS. Hoechst 33342 $(0.2 \mathrm{~g} / \mathrm{ml}$, Calbiochem $)$ was used to visualize nucleus. The anti-Flag (Cell Signalling, M2) primary antibody was used to detect 3XFLAG-SpCas9. Imaging was performed on a DeltaVision RT system (Applied Precision) with an Olympus IX71 microscope using Softworx software (Applied Precision).

For immunoblotting, cells were washed twice with PBS and lysed in lysis buffer $(10 \mathrm{mM}$ Tris-Cl pH 7.5, $150 \mathrm{mM}$ $\mathrm{NaCl}, 5 \mathrm{mM}$ EDTA, 0.1 \% SDS, 1\%Triton X-100, $1 \%$ deoxycholate) supplemented with $1 \mathrm{mM}$ PMSF (Sigma) and protease inhibitor cocktail (Roche) for $30 \mathrm{~min}$. Lysates were centrifuged and the supernatant was boiled with Laemmli buffer. SDS-PAGE was performed as previously 
described [31]. The membranes were blocked in $5 \%$ nonfat milk in TBS-T and anti- $\alpha$-tubulin (Sigma, T-9026), anti-Flag (Cell Signalling, M2) primary antibodies were used with appropriate secondary antibodies.

\section{Cell culture}

hTERT-RPE1 cells were cultured in Dulbecco's Modified Eagle's Medium (DMEM) F12 (Gibco) supplemented with heat inactivated $10 \%$ fetal bovine serum (FBS), $100 \mathrm{U} / \mathrm{ml}$ penicillin and $100 \mathrm{mg} / \mathrm{ml}$ streptomycin. HCT116 cells were cultured in McCoy's 5A (Gibco) medium supplemented with $10 \% \mathrm{FBS}, 100 \mathrm{U} / \mathrm{ml}$ penicillin and $100 \mathrm{mg} / \mathrm{ml}$ streptomycin. All cell lines were cultured at $37{ }^{\circ} \mathrm{C}$ with $5 \% \mathrm{CO}_{2}$.

\section{Ethics statement}

No ethics approval was required for this study.

\section{Availability of supporting data}

The supporting sequencing files for Figs. 3, 4 and 5 are attached as supplementary files with the manuscript.

\section{Additional files}

Additional file 1: Figure S1. Schematic illustration of genome editing by engineered nucleases. Engineered nucleases like zinc finger nucleases (ZFN), transcription activator-like effector nuclease (TALEN), and the RNA-guided clustered regularly interspaced short palindromic repeats (CRISPR)-Cas9 nuclease systems are targeted to specific nucleotide sequences to cause double strand break (DSB). Cells can repair this DSB either by high-fidelity homologous recombination (HR) and/or error-prone non-homologous end-joining (NHEJ) approaches [16]. Homologous recombination event can be manipulated to cause specific genome editing by introducing a donor template. On the other hand, NHEJ might disrupt gene function due to the random insertion or deletion of nucleotides of varying length (PDF $432 \mathrm{~kb}$ )

Additional file 2: Figure S2. Strategy for Cas9-mediated generation of hCDC14A knockout RPE1 cells. (A) Workflow for the construction of RPE1 hCDC14A-KO cell line. pCW-Cas9 plasmid containing doxycycline (Dox) inducible spCas9 was lentivirally integrated into RPE1 FRT/T-Rex cells. (B, C) Successful expression and nuclear localization of Cas9 was confirmed by indirect-immunofluorescence (B) and western blotting (C). (D) Junction PCR with forward primer in NeoR cassette and reverse primer in the genome outside homology arm (as in Fig. 1a) confirmed successful targeting and insertion of the selection marker. Exon skipping was confirmed by RT-PCR (primers as in Fig. 3a). Presence of both wild type and exon-skipped RNA indicated the targeting of single allele in clones 1 and 20. (PDF $1952 \mathrm{~kb}$ )

\section{Abbreviations \\ CRISPR: Clustered regularly interspaced short palindromic repeats; \\ CDS: Coding sequence; Cas9: CRISPR-associated protein-9 nuclease; \\ CDKs: Cyclin-dependent kinases; DSB: Double strand breaks; \\ HA: Homologous arm; HR: Homologous recombination; hTERT-RPE1: Human telomerase reverse transcription-immortalized retinal pigment epithelial cells; hCDC14: Human cell division cycle 14; NHEJ: Non-homologous end joining; RT-PCR: Reverse transcription polymerase chain reaction; TALEN: Transcription activator-like effector nuclease; ZFN: Zinc-finger nuclease.}

\section{Competing interests}

The authors declare that they have no competing interests.

\section{Authors' contributions}

Conceived and designed the experiment: ES, BU, NPC. Performed the experiment: BU, NPC, MP. Analyzed the data: BU, NPC, MP; Wrote the paper: $\mathrm{BU}, \mathrm{ES}$. All authors read and approve the final manuscript.

\section{Acknowledgement}

We thank Dr. Gislene Pereira for scientific discussion and helpful suggestions. We also thank Ana Pastor Peidro for help during Southern Blot hybridization and Berati Cerikan for critical discussions on the project. We acknowledge Deutsche Forschungsgemeinschaft grant Schi 295/3-2 for funding. The funders had no role in study design, collection, analysis, and interpretation of data; in writing of the manuscript; and in the decision to submit the manuscript for publication.

\section{Author details}

'Zentrum für Molekulare Biologie der Universität Heidelberg (ZMBH), DKFZ-ZMBH Allianz, Im Neuenheimer Feld 282, 69120 Heidelberg, Germany. ${ }^{2}$ The Hartmut Hoffmann-Berling International Graduate School of Molecular and Cellular Biology (HBIGS), University of Heidelberg, Heidelberg 69120, Germany.

Received: 9 August 2015 Accepted: 10 December 2015

Published online: 21 December 2015

\section{References}

1. Morgan DO. Cyclin-dependent kinases: engines, clocks, and microprocessors. Annu Rev Cell Dev Biol. 1997;13:261-91.

2. Stegmeier F, Amon A. Closing mitosis: the functions of the Cdc14 phosphatase and its regulation. Annu Rev Genet. 2004;38:203-32.

3. Li L, Ljungman M, Dixon JE, Ljungmann M, Dixon JE. The Human Cdc14 Phosphatases Interact with and Dephosphorylate the Tumor Suppressor Protein p53. J Biol Chem. 2000;275:2410-4.

4. Rosso L, Marques AC, Weier M, Lambert N, Lambot M-AA, Vanderhaeghen $P$, et al. Birth and rapid subcellular adaptation of a hominoid-specific CDC14 protein. PLoS Biol. 2008:6:e140.

5. Gray CH, Good VM, Tonks NK, Barford D. The structure of the cell cycle protein $\mathrm{Cdc} 14$ reveals a proline-directed protein phosphatase. EMBO J. 2003:22:3524-35.

6. Cho HP, Liu Y, Gomez M, Dunlap J, Wang Y, Tyers M. The dual-specificity phosphatase CDC14B bundles and stabilizes microtubules the dualspecificity phosphatase CDC14B bundles and stabilizes microtubules †. Mol Cell Biol. 2005:25:4541-51.

7. Mocciaro A, Schiebel E. Cdc14: a highly conserved family of phosphatases with non-conserved functions? J Cell Sci. 2010:123(Pt 17):2867-76.

8. Mailand N, Falck J, Lukas C, Syljuåsen RG, Welcker M, Bartek J, et al. Rapid destruction of human Cdc25A in response to DNA damage. Science. 2000; 288:1425-9.

9. Tumurbaatar I, Cizmecioglu O, Hoffmann I, Grummt I, Voit R. Human Cdc14B promotes progression through mitosis by dephosphorylating Cdc25 and regulating Cdk1/Cyclin B activity. PLoS One. 2011;6:e14711.

10. Bassermann F, Frescas D, Guardavaccaro D, Busino L, Peschiaroli A, Pagano M. The Cdc14B-Cdh1-PIk1 axis controls the G2 DNA-damage-response checkpoint Cell. 2008:134:256-67.

11. Berdougo E, Nachury MV, Jackson PK, Jallepalli PV. The nucleolar phosphatase Cdc14B is dispensable for chromosome segregation and mitotic exit in human cells. Cell Cycle. 2008;7:1184-90.

12. Mocciaro A, Berdougo E, Zeng K, Black E, Vagnarelli $P$, Earnshaw W, et al. Vertebrate cells genetically deficient for $\mathrm{Cdc14A}$ or $\mathrm{Cdc} 14 \mathrm{~B}$ retain DNA damage checkpoint proficiency but are impaired in DNA repair. J Cell Biol. 2010;189:631-9.

13. Ovejero S, Ayala P, Bueno A, Sacristán MP. Human Cdc14A regulates Wee1 stability by counteracting CDK-mediated phosphorylation. Mol Biol Cell. 2012;23:4515-25.

14. Guillamot M, Manchado E, Chiesa M, Gómez-López G, Pisano DG, Sacristán MP, et al. Cdc14b regulates mammalian RNA polymerase $\|$ and represses cell cycle transcription. Sci Rep. 2011:1:189.

15. Ran FA, Hsu PPD, Wright J, Agarwala V, Scott D a, Zhang F. Genome engineering using the CRISPR-Cas9 system. Nat Protoc. 2013:8:2281-308.

16. Sonoda E, Hochegger $H$, Saberi A, Taniguchi $Y$, Takeda S. Differential usage of non-homologous end-joining and homologous recombination in double strand break repair. DNA Repair (Amst). 2006:5:1021-9. 
17. Valentine CR. The association of nonsense codons with exon skipping Mutat Res Rev Mutat Res. 1998;411:87-117.

18. Bremmer SC, Hall H, Martinez JS, Eissler CL, Hinrichsen TH, Rossie S, et al. Cdc14 phosphatases preferentially dephosphorylate a subset of cyclindependent kinase (Cdk) sites containing phosphoserine. J Biol Chem. 2012; 287:1662-9.

19. Hall MC, Jeong DE, Henderson JT, Choi E, Bremmer SC, lliuk AB, et al. Cdc28 and Cdc14 control stability of the anaphase-promoting complex inhibitor Acm1. J Biol Chem. 2008;283:10396-407.

20. Langeberg LK, Scott JD. Signalling scaffolds and local organization of cellular behaviour. Nat Rev Mol Cell Biol. 2015;16:232-44.

21. Zhu L, Zhang Y, Zhang W, Yang S, Chen J-Q, Tian D. Patterns of exon-intron architecture variation of genes in eukaryotic genomes. BMC Genomics. 2009;10:47.

22. Sakharkar MK, Chow VTK, Kangueane P. Distributions of exons and introns in the human genome. In Silico Biol. 2004;4:387-93.

23. Panic M, Hata S, Neuner A, Schiebel E. The centrosomal linker and microtubules provide dual levels of spatial coordination of centrosomes. PLoS Genet. 2015;11:e1005243.

24. Doyon Y, Choi VM, Xia DF, Vo TD, Gregory PD, Holmes MC. Transient cold shock enhances zinc-finger nuclease-mediated gene disruption. Nat Methods. 2010;7:459-60.

25. Hsu PD, Scott D a, Weinstein J a, Ran FA, Konermann S, Agarwala V, et al. DNA targeting specificity of RNA-guided Cas9 nucleases. Nat Biotechnol. 2013;31:827-32.

26. Mali P, Yang L, Esvelt KM, Aach J, Guell M, Dicarlo JE, et al. RNA-guided human genome engineering via Cas9. Science. 2013;339:823-6.

27. Wang T, Wei JJ, Sabatini DM, Lander ES. Genetic screens in human cells using the CRISPR-Cas9 system. Science. 2014;343:80-4.

28. Southern E. Southern blotting. Nat Protoc. 2006;1:518-25.

29. Zhang Y, Ba Y, Liu C, Sun G, Ding L, Gao S, et al. PGC-1alpha induces apoptosis in human epithelial ovarian cancer cells through a PPARgamma-dependent pathway. Cell Res. 2007;17:363-73.

30. Carbery ID, Ji D, Harrington A, Brown V, Weinstein EJ, Liaw L, et al. Targeted genome modification in mice using zinc-finger nucleases. Genetics. 2010; 186:451-9.

31. Laemmli UK. Cleavage of structural proteins during the assembly of the head of bacteriophage T4. Nature. 1970;227:680-5.

\section{Submit your next manuscript to BioMed Central and we will help you at every step:}

- We accept pre-submission inquiries

- Our selector tool helps you to find the most relevant journal

- We provide round the clock customer support

- Convenient online submission

- Thorough peer review

- Inclusion in PubMed and all major indexing services

- Maximum visibility for your research

Submit your manuscript at www.biomedcentral.com/submit 